\title{
A RANDOM MULTIPLE-ACCESS PROTOCOL WITH SPATIAL INTERACTIONS
}

\author{
CHARLES BORDENAVE, * University of California \\ SERGUEI FOSS, ${ }^{* *}$ Heriot-Watt University and Sobolev Institute of Mathematics \\ VSEVOLOD SHNEER, ${ }^{* * *}$ Eindhoven University of Technology and EURANDOM
}

\begin{abstract}
We analyse an ALOHA-type random multiple-access protocol where users have local interactions. We show that the fluid model of the system workload satisfies a certain differential equation. We obtain a sufficient condition for the stability of this differential equation and deduce from that a sufficient condition for the stability of the protocol. We discuss the necessary condition. Furthermore, for the underlying Markov chain, we estimate the rate of convergence to the stationary distribution. Then we establish an interesting and unexpected result showing that the main diagonal is locally unstable if the input rate is sufficiently small. Finally, we consider two generalisations of the model.
\end{abstract}

Keywords: ALOHA protocol; spatial interaction; stability of processes; fluid limits

2000 Mathematics Subject Classification: Primary 60K25; 90B15

Secondary $68 \mathrm{R} 10$

\section{Introduction and stability result}

\subsection{A spatial ALOHA}

We consider a random spatial service system governed by an ALOHA-type algorithm. More precisely, time is slotted, i.e. during each of the time slots $n=1,2, \ldots$ a random number, $\xi_{n}$, of users arrive in the system, and at the beginning of each time slot every user in the system requires service (transmission) with a certain (transmission) probability independently of all other users. The sequence $\left\{\xi_{n}\right\}$ is assumed to be independent and identically distributed (i.i.d.).

The ALOHA multiple-access algorithm was first proposed by Abramson [1]. The slotted scheme was introduced by Roberts [15]. We consider the latter setting. In the conventional centralised slotted ALOHA model, there is a single server. At the beginning of time slot $n$, the total number, $W_{n}$, of users in the system is known, and each of them asks for service (transmission) with probability $1 / W_{n}$ independently of all other users. If two or more users attempt transmission simultaneously then the transmissions collide and the users remain in the system and try to transmit again later. All service times are equal to 1 and the server is free at the beginning of any time slot. It is easy to show that, for this system, the maximum throughput is equal to $\mathrm{e}^{-1}$. Furthermore, the Markov chain $\left\{W_{n}\right\}$ is positive recurrent if $\mathrm{E} \xi_{1}<\mathrm{e}^{-1}$ and transient if $\mathrm{E} \xi_{1}>\mathrm{e}^{-1}$.

\footnotetext{
Received 13 December 2006; revision received 11 June 2009.

* Current address: CNRS UMR 5219, Institut de Mathématiques de Toulouse, 118 route de Narbonne, F-31062 Toulouse, France.

** Postal address: Department of Actuarial Mathematics and Statistics, Heriot-Watt University, Edinburgh EH14 4AS, UK.

*** Postal address: EURANDOM, PO Box 513, 5600 MB Eindhoven, The Netherlands.

Email address: shneer@eurandom.tue.nl
} 
When information on the number, $W_{n}$, of users is unavailable, various decentralised adaptive algorithms have been introduced and studied. Algorithms of this type use information about what has occurred in the previous time slot: either a conflict or a successful transmission or no attempted transmissions. More precisely, let $B_{n}$ be the number of users trying to transmit during time slot $n$. In a decentralised algorithm, only the values of $\min \left\{B_{n}, 2\right\}$ are available at time $n+1$. Assuming that at any time slot $n$ the transmission probability is the same for all users, Hajek [11] proved that if $\left\{\xi_{n}\right\}$ is i.i.d. with a finite exponential moment then $\mathrm{E} \xi_{n}<\mathrm{e}^{-1} \approx 0.37$ is still necessary and sufficient for the existence of a stable algorithm. Mikhaulov [14] generalised this result by weakening the exponential moment assumption to the requirement for only the second moment to exist, while Foss [9] generalised it further by dropping this as well as the independence assumption. We also refer to Ephremides and Hajek [6] for a survey which includes, in particular, results in this direction.

The model described above ignores the network's spatial diversity. In this paper we present a new model which allows the possibility for interaction between users to occur only when the distance between these users is small. A limited spatial interaction is a common feature of wireless networks and is thus very important for practical consideration.

In this paper we consider only spatial centralised schemes (protocols) where the total number of users in a neighbourhood is known. The study of decentralised protocols is a subject of our future research. Our main result establishes the stability of the model under consideration. For this, we use the fluid approximation approach. We show that the state is repelled by the boundary, and in the interior, the Euclidean norm turns out to be a Lyapunov function for the fluid model. Other results of the paper include convergence rates to stationarity and local (in)stability.

The remainder of the paper is organised as follows. The rest of this introduction is devoted to the description of the model and the statement of our main stability result. In Section 2 we prove that fluid limits of the workload in the system satisfy a certain differential equation. Section 3 is devoted to the analysis of the behaviour of fluid limits on the boundary of the positive orthant. In Section 4 we present a proof of our main stability result and formulate one of its possible generalisations. Section 5 deals with rates of convergence towards the steady state. Sections 6 and 7 contain some interesting results on the behaviour of solutions to the differential equation. Finally, in Section 8 we conclude with some extensions of our model, which are in a certain sense more applicable to real systems. In particular, these extensions include systems where various changes in environmental conditions may result in changes in the radius and/or direction of interference between the message transmissions.

\subsection{Model description}

Let $g=(\mathcal{V}, \mathcal{E})$ be an undirected graph with a finite set of vertices, say $\mathcal{V}=\{1, \ldots, K\}$. We suppose that $g$ is connected. For the graph $g$, we use the standard notion of the graph distance. For $i \in \mathcal{V}$, let $V_{i}=\{i\} \cup\{j \in \mathcal{V}$ : such that $(i, j) \in \mathcal{E}\}$ be the neighbourhood of a vertex $i$, i.e. the set of vertices at a maximum distance of 1 from $i$ in the graph. In the particular case where all vertices have the same number of neighbours, we denote by $V$ the cardinality of $V_{i}$, $\operatorname{card}\left(V_{i}\right)=V$.

We now introduce a service system with spatial (neighbourhood) interactions associated with the graph $g$. We assume that time is slotted, i.e. arrivals and services may occur only at times $n=1,2, \ldots$ Suppose that there is a service station at each vertex of $g$. The arrival process is denoted by $A=(A(n))_{n \in \mathbb{N}}$, where $A(n)=\left(A_{1}(n), \ldots, A_{K}(n)\right) \in \mathbb{N}^{K}$, and $A_{i}(n)$ is the number of users arriving at time $n$ at a vertex $i$. For $s<t$, denote by $A(s, t)=\sum_{n=\lceil s\rceil}^{\lceil t\rceil-1} A(n)$ 
the vector representing the number of users arriving between times $s$ and $t-$. Here $\lceil t\rceil$ denotes the smallest integer larger than or equal to $t$. We suppose that $(A(n))$ is an i.i.d. sequence (while the coordinates of the vectors may be dependent). We also suppose that $\mathrm{E} A_{i}(n)=\lambda_{i}>0$ for $i=1, \ldots, K$. If values of $\lambda_{i}$ do not depend on $i$ then we write $\lambda_{i}=\lambda, i=1, \ldots, K$.

Let $W(n)=\left(W_{1}(n), \ldots, W_{K}(n)\right) \in \mathbb{R}_{+}^{K}$, where $W_{i}(n)$ is the number of users at vertex $i$ at time $n$. At time $n$, a user at vertex $i$ attempts transmission independently of the other users with probability $1 / \sum_{j \in V_{i}} W_{j}(n)$. This user receives service if he is the only user attempting transmission in $V_{i}$ at time $n$. We suppose that all service times are equal to 1 and that any user leaves the system immediately upon service completion. Let $N_{i}(n)$ be the number of users attempting transmission at time $n$ at vertex $i$. Then $N_{i}(n)$ is a binomial random variable with parameters $\left(W_{i}(n), 1 / \sum_{j \in V_{i}} W_{j}(n)\right)$ and $\left\{N_{i}(n), 1 \leq i \leq K\right\}$ are independent variables conditioned on $W(n)$. The sequence $\{W(n)\}$ forms a time-homogeneous, irreducible Markov chain, which satisfies the recursion

$$
W_{i}(n)=W_{i}(n-1)+A_{i}(n)-I\left(N_{i}(n-1)=1\right) \prod_{j \in V_{i} \backslash\{i\}} I\left(N_{j}(n-1)=0\right),
$$

where $I(\cdot)$ denotes the indicator function.

To explicitly show the dependence of $W(n)$ on the initial condition $W(0)=x$, we sometimes write $W^{x}(n)$.

If $x_{i}>0$ then the $i$ th component of the drift vector is given by the expression

$$
\begin{aligned}
& \mathrm{E}\left[W_{i}(1)-W_{i}(0) \mid W(0)=x\right] \\
& \quad=\lambda_{i}-\frac{x_{i}}{\sum_{k \in V_{i}} x_{k}}\left(1-\frac{1}{\sum_{k \in V_{i}} x_{k}}\right)^{x_{i}-1} \prod_{j \in V_{i} \backslash\{i\}}\left(1-\frac{1}{\sum_{k \in V_{j}} x_{k}}\right)^{x_{j}},
\end{aligned}
$$

and if $x_{i}=0$ then $\mathrm{E}\left[W_{i}(1)-W_{i}(0) \mid W(0)=x\right]=\lambda_{i}$.

We rewrite the expression for the drift vector as

$$
\mathrm{E}[W(1)-W(0) \mid W(0)=x]=\underline{\lambda}-G(x) .
$$

Here $\underline{\lambda}$ is a $K$-dimensional vector with its $i$ th component being equal to $\lambda_{i}$ and $G(x)=$ $\left(G_{1}(x), \ldots, G_{K}(x)\right)$ is a function from $\mathbb{R}^{K}$ to $\mathbb{R}^{K}$ defined by

$$
G_{i}(x)= \begin{cases}\frac{x_{i}}{\sum_{k \in V_{i}} x_{k}}\left(1-\frac{1}{\sum_{k \in V_{i}} x_{k}}\right)^{x_{i}-1} \prod_{j \in V_{i} \backslash\{i\}}\left(1-\frac{1}{\sum_{k \in V_{j}} x_{k}}\right)^{x_{j}} & \text { if } x_{i}>0, \\ & \text { if } x_{i}=0 .\end{cases}
$$

For $x \in \mathbb{R}^{K}$, we define $\phi_{i}(x)=x_{i} / \sum_{j \in V_{i}} x_{j}$. Let $\phi(x)=\left(\phi_{1}(x), \ldots, \phi_{K}(x)\right)$. Note that $G_{i}$ is bounded from above by 1 and if $\sum_{k \in V_{i}} x_{k}>0$ then

$$
\lim _{t \rightarrow+\infty} G_{i}(t x)=\widetilde{G}_{i}(x)=\phi_{i}(x) \exp \left\{-\sum_{j \in V_{i}} \phi_{j}(x)\right\} .
$$

In particular, $\widetilde{G}_{i}$ is a homogeneous function of order 0 , i.e. $\widetilde{G}_{i}(c x)=\widetilde{G}_{i}(x)$ for any $c>0$.

We now comment on the model. In this paper we mostly consider the symmetric case, where $\lambda_{i}=\lambda$ for all $i=1, \ldots, K$ and the graph $g$ is $(V-1)$-regular: the cardinality of $V_{i}$ is equal to $V$ for all $i$. Note that, even in this case, the graph $g$ is not necessarily completely symmetric. 
Note also that the system is not monotone. Indeed, $x \leq y$ (componentwise) does not imply that $W^{y}(1)$ stochastically dominates $W^{x}(1)$. Neither is the system monotone with respect to the graph structure: if $G_{1}$ is embedded into $G_{2}$, this does not imply that the workload process built on graph $G_{1}$ is stochastically dominated by the workload built on graph $G_{2}$.

We also present a number of generalisations in the nonsymmetric case. In particular, using methods suggested recently in [18], we formulate in Remark 3 sufficient conditions for the stability of the system with space-inhomogeneous input. Some other generalisations of the model are proposed in Section 8.

\subsection{Stability result}

We first explain the intuition hidden behind the result. Consider the symmetric case, $\lambda_{i}=\lambda$ and $\operatorname{card}\left(V_{i}\right)=V, i=1, \ldots, K$.

The access protocol favours an equilibrium of the workload in the network: assume that the workload at node $i$ is much larger than the workload in its neighbouring nodes, $V_{i} \backslash\{i\}$. Then $\phi_{i}(x)$ is close to 1 , whereas $\phi_{j}(x)$ is close to 0 for all the other nodes $j$ in $V_{i}$. Thus, the workload at node $j$ in $V_{i}$ will tend to get closer to the workload at node $i$. From this balance mechanism we can guess that the diagonal $\Delta=\left\{x \in \mathbb{R}^{K}: x_{1}=x_{2}=\cdots=x_{K}\right\}$ is an attractive set.

If the workload vector belongs to the diagonal: $W(0)=c \mathbf{1}$, where $c \in \mathbb{N}$ and $\mathbf{1}=(1, \ldots, 1)$, then we obtain

$$
\mathrm{E}[W(1)-W(0) \mid W(0)=c \mathbf{1}]=\left(\lambda-\frac{1}{V}\left(1-\frac{1}{V c}\right)^{V c-1}\right) \mathbf{1} .
$$

Hence, as $c$ tends to $\infty$, the drift vector converges to $\left(\lambda-\mathrm{e}^{-1} / V\right) \mathbf{1}$.

We finally end up with the conjecture that if $\lambda<\mathrm{e}^{-1} / V$ then the Markov chain $W$ is ergodic. This conjecture is clearly true for the fully isolated graph and for the complete graph.

The reasons that led to this conjecture appear to be wrong (this will follow from the results of Sections 6 and 7, which show that in general the main diagonal is not attractive). However, the conjecture itself is true and we can formulate our main stability result that will be proved in Section 4.

Theorem 1. Assume that the model is symmetric.

(i) If $\lambda<\mathrm{e}^{-1} / V$ then the Markov chain $W$ is positive recurrent.

(ii) Assume further that

$$
\mathrm{P}\left(A_{1}(1)=A_{2}(1)=\cdots=A_{K}(1)=0\right)>0 .
$$

Then $W$ is ergodic, i.e. there exists a unique stationary distribution and the distribution of $W_{n}$ converges to the stationary one in the total variation norm.

Our proof of (i) is based on the fluid approximation approach. We will show that all fluid limits satisfy a certain differential equation and then proceed with the analysis of that equation. The proof of (ii) is standard: the state $\mathbf{0}=(0,0, \ldots, 0)$ is achievable from any other state and from itself. So, the state $\mathbf{0}$ is positive recurrent (due to (i)) and the Markov chain $W$ is aperiodic. The ergodicity follows.

Our heuristics also suggest that if $\lambda>\mathrm{e}^{-1} / V$ then $W$ is transient. Corollary 4 in Section 6 is a partial result which corroborates this intuition. 


\section{Fluid approximation method}

This section deals with a general (not necessarily symmetric) graph.

\subsection{General properties}

In what follows, we endow $\mathbb{R}^{K}$ with the $L^{1}$-norm: $|x|=\sum_{k=1}^{K}\left|x_{k}\right|$. Let $\left(x^{n}\right), n \in \mathbb{N}$, be a sequence in $\mathbb{N}^{K}$ such that $\lim _{n}\left|x^{n}\right|=\infty$. For $t \in[0, T]$, we define

$$
X^{n}(t)=\frac{W^{x^{n}}\left(\left\lceil\left|x^{n}\right| t\right\rceil\right)}{\left|x^{n}\right|} .
$$

To simplify the notation, for $t \in \mathbb{R}_{+}$, we set $W(t)=W(\lceil t\rceil)$.

Let $\mathbb{D}\left([0, T], \mathbb{R}^{K}\right)$ denote the space of càdlàg functions from $[0, T]$ to $\mathbb{R}^{K}$ endowed with the usual Skorokhod topology. This means that the distance between the functions $f_{1}$ and $f_{2}$ is defined by

$$
d_{T}\left(f_{1}, f_{2}\right)=\inf \sup _{t \in[0, T]}\left\{|g(t)-t|+\rho\left(f_{1}(g(t)), f_{2}(t)\right)\right\}
$$

where $\rho$ is the $L^{1}$-metric in $\mathbb{R}^{K}$ and the outer infimum is taken over all monotone continuous functions $g:[0, T] \rightarrow[0, T]$ such that $g(0)=0$ and $g(T)=T$. Denote by $\mathbb{D}([0, \infty))$ the space of $\mathbb{R}^{K}$-valued càdlàg functions on $[0, \infty)$ with the metric

$$
d\left(f_{1}, f_{2}\right)=\sum_{T=1}^{\infty} 2^{-T} \frac{d_{T}\left(f_{1}, f_{2}\right)}{1+d_{T}\left(f_{1}, f_{2}\right)} .
$$

Note that $X^{n} \in \mathbb{D}\left([0, T], \mathbb{R}^{K}\right)$ for all $n$.

Lemma 1. (i) For any sequence $x^{n}$ such that $\left|x^{n}\right| \rightarrow \infty$, the family $\left\{\left(X^{n}\right), n \in \mathbb{N}\right\}$ almost surely (a.s.) has a compact closure in the Skorokhod topology, and any accumulation point $z$ of $\left\{X_{n}\right\}$ is a.s. continuous.

(ii) Function $z$ is Lipschitz with the constant $\max \left\{\sum_{i=1}^{K} \lambda_{i}, K\right\}$.

Proof. (i) We can easily obtain a proof of this statement along the lines of the proof of [3, Theorem 4.1] or [17, Theorem 7.1]. Formally, the proofs of the mentioned theorems are given for multiclass networks. However, as pointed out in [10], the tightness of such families holds under weaker conditions (see [10, Assumption 2.19]).

(ii) Since the $G_{i}$ are bounded by 1 from above,

$$
\begin{aligned}
\left|X^{n}(t)-X_{i}^{n}(s)\right| & \leq \max \left\{\frac{\left|A\left(s\left|x^{n}\right|, t\left|x^{n}\right|\right)\right|}{\left|x^{n}\right|}, \frac{K\left|x^{n}\right|\left(t-s+1 /\left|x^{n}\right|\right)}{\left|x^{n}\right|}\right\} \\
& \leq \max \left\{\frac{1}{\left|x^{n}\right|} \sum_{k=\left\lceil\left|x^{n}\right| s\right\rceil}^{\left\lfloor\left|x^{n}\right| t\right\rfloor} B_{k}, K\left(t-s+\frac{1}{\left|x^{n}\right|}\right)\right\},
\end{aligned}
$$

where $B_{k}$ is the total number of arrivals at time $k$ and by $\lfloor z\rfloor$ we denote the largest integer which smaller than or equal to $z$. Sequence $\left\{B_{k}\right\}_{k \in \mathbb{N}}$ consists of i.i.d. random variables with $\mathrm{E} B_{k}=K \underline{\lambda}$. By the law of large numbers, the result now follows if we let $n \rightarrow \infty$.

Definition 1. Any accumulation (in the Skorokhod topology) point $z$ of the sequence $X^{n}$ is a fluid limit. The collection of all fluid limits is called the fluid model. 
It follows from the definition of $X^{n}$ and $z$ that $|z(0)|=1$ and that $z_{i}(t) \geq 0$ for all $i=$ $1, \ldots, K$ and all $t$.

Corollary 1. The sample-path trajectories of fluid limits are self-similar. More precisely, for any fluid limit $z$ and any $u>0$ such that $\mathrm{P}(|z(u)|>0)>0$, the random process $\{\tilde{z}(t), t \geq 0\}$ with conditional distribution

$$
\mathrm{P}(\widetilde{z}(t) \in \cdot)=\mathrm{P}\left(\frac{z(u+t)}{|z(u)|} \in \cdot \mid z(u)\right)
$$

is also a fluid limit on the event $|z(u)|>0$.

This result may be obtained along the lines of the proof of Lemma 6.1 of [17]. However, the remark given in the proof of Lemma 1(i) also applies here.

Definition 2. We say that the fluid model is stable if there exist a deterministic time $t_{0}$ and a $\varepsilon \geq 0$ such that $|z(t)| \leq \varepsilon$ a.s., $t \geq t_{0}$, for all fluid limits $z$. Owing to the self-similarity of fluid limits, this is equivalent to saying that there exists a deterministic time $t$ such that $|z(t)|=0$ a.s. for all fluid limits $z$.

This definition of fluid stability has become standard and appears in most papers dealing with the fluid approximation method.

\subsection{Fluid model criterion for stability}

In this subsection we formulate a stability theorem for fluid limits which will imply Theorem 1(i).

Lemma 2. If the fluid model is stable then $W$ is positive recurrent.

Proof. Again, we can obtain a proof of this assertion by following the lines of the proofs in [3] and [17], which are given for multiclass networks.

By Lemma 2, Theorem 1(i) will follow from the next statement.

Theorem 2. If $\lambda<\mathrm{e}^{-1} / V$ then the fluid model is stable.

Our proof of Theorem 2 is given in Section 4.

\subsection{Fluid limit evolution equation}

In what follows we write

$$
\varphi_{i}(t)=\phi_{i}(z(t))=\frac{z_{i}(t)}{\sum_{j \in V_{i}} z_{j}(t)} .
$$

Theorem 3. Take any fluid limit $z$ and fix $t \geq 0$. Assume that $\sum_{j \in V_{i}} z_{j}(t)>0$ for all $i$. Then $z_{i}(t)$ is differentiable at point $t$ if $t>0$ and has a right derivative at point $t$ if $t=0$. Moreover,

$$
z_{i}^{\prime}(t)=\lambda_{i}-\varphi_{i}(t) \exp \left\{-\sum_{j \in V_{i}} \varphi_{j}(t)\right\}=\lambda_{i}-\widetilde{G}_{i}\left(z_{i}(t)\right),
$$

where $z_{i}^{\prime}(t)$ is the (right) derivative. 
Under the assumptions of Theorem 3, (2) admits a unique solution.

Fluid limits with an initial condition on the boundary $\left(\sum_{j \in V_{i}} z_{j}(0)=0\right.$ for some $\left.i\right)$ are analysed in Section 3.

Before proving Theorem 3, we need the following technical result.

Lemma 3. There exists a $C>0$ such that $\left|G_{i}(x)-\widetilde{G}_{i}(x)\right| \leq \min \left(1, C / x_{i}\right)$ if $x_{i} \geq 2$.

Proof. Using the fact that $\left|\exp \left\{-y_{1}\right\}-\exp \left\{y_{2}\right\}\right| \leq\left|y_{1}-y_{2}\right|$ for all $y_{1}, y_{2} \geq 0$, we obtain

$$
\begin{aligned}
\left|G_{i}(x)-\widetilde{G}_{i}(x)\right| \leq & \left|\ln \left(1-\frac{1}{\sum_{k \in V_{i}} x_{k}}\right)\right| \\
& +\left|\sum_{j \in V_{i}}\left(x_{j} \ln \left(1-\frac{1}{\sum_{k \in V_{j}} x_{k}}\right)+\frac{x_{j}}{\sum_{k \in V_{j}} x_{k}}\right)\right| .
\end{aligned}
$$

For every $j$, define $y_{j}=1 / \sum_{k \in V_{j}} x_{k}$. From the inequality $|\ln (1-y)+y| \leq y^{2} / 2(1-y)^{2}$ for $y \in(0,1)$ we obtain

$$
\left|G_{i}(x)-\widetilde{G}_{i}(x)\right| \leq y_{i}+\frac{y_{i}^{2}}{2\left(1-y_{i}\right)^{2}}+\sum_{j \in V_{i}} \frac{x_{j} y_{j}^{2}}{2\left(1-y_{j}\right)^{2}} .
$$

The statement of the lemma now follows from the inequalities

$$
y_{j} \leq \frac{1}{x_{i}}, \quad x_{j} y_{j} \leq 1, \quad \text { and } \quad y_{j} \leq \frac{1}{2}, \quad \text { for all } j \in V_{i} .
$$

Proof of Theorem 3. Two cases are possible: $z_{i}(t)>0$ or $z_{i}(t)=0$ and $\sum_{j \in V_{i}} z_{j}(t)>0$. We treat these two cases separately.

Case 1: $z_{i}(t)>0$. Assume that $t=0$ (the result for an arbitrary $t$ follows from the self-similarity of fluid limits). Let $x=z(0)$. Suppose that $s<x_{i}$. Let $k \leq\left|x^{n}\right| s$. Then $W_{i}^{x^{n}}(k) \geq x_{i}^{n}-k \geq\left|x^{n}\right|\left(x_{i}^{n} /\left|x^{n}\right|-s\right)$. Hence, $W_{i}^{x^{n}}(k) \geq 2$ for $k \leq\left|x^{n}\right| s$, for large enough $n$.

We need to show that $\lim _{s \rightarrow 0}\left(z_{i}(s)-z_{i}(0)\right) / s=\lambda_{i}-\widetilde{G}_{i}(z(0))$. Consider the decomposition

$$
\begin{aligned}
X_{i}^{n}(s)-X_{i}^{n}(0)= & \frac{1}{\left|x^{n}\right|} \sum_{k=0}^{\left\lfloor\left|x^{n}\right| s\right\rfloor-1}\left(W_{i}^{x^{n}}(k+1)-W_{i}^{x^{n}}(k)\right) \\
= & \frac{1}{\left|x^{n}\right|} \sum_{k=0}^{\left\lfloor\left|x^{n}\right| s\right\rfloor-1} \mathrm{E}\left[W_{i}^{x^{n}}(k+1)-W_{i}^{x^{n}}(k) \mid W^{x^{n}}(k)\right] \\
& +\frac{1}{\left|x^{n}\right|} \sum_{k=0}^{\left\lfloor\left|x^{n}\right| s\right\rfloor-1}\left(W_{i}^{x^{n}}(k+1)-\mathrm{E}\left[W_{i}^{x^{n}}(k+1) \mid W_{i}^{x^{n}}(k)\right]\right) \\
= & \frac{1}{\left|x^{n}\right|} \sum_{k=0}^{\left\lfloor\left|x^{n}\right| s\right\rfloor-1}\left(\lambda_{i}-G_{i}\left(W^{x^{n}}(k)\right)\right)+\frac{1}{\left|x^{n}\right|} \sum_{k=1}^{\left\lfloor\left|x^{n}\right| s\right\rfloor} D_{k}^{n},
\end{aligned}
$$

where

$$
D_{k}^{n}=W_{i}^{x^{n}}(k)-\mathrm{E}\left[W_{i}^{x^{n}}(k) \mid W^{x^{n}}(k-1)\right]=A_{i}(k)-\lambda_{i}+q_{i}(k)-\mathrm{E}\left[q_{i}(k) \mid W^{x^{n}}(k-1)\right]
$$


with $q_{i}(k)=I\left(N_{i}(k-1)=1\right) \prod_{j \in V_{i} \backslash\{i\}} I\left(N_{j}(k-1)=0\right)$. We have

$$
\frac{1}{\left|x^{n}\right|} \sum_{k=1}^{\left\lfloor\left|x^{n}\right| s\right\rfloor}\left(A_{i}(k)-\lambda_{i}\right) \rightarrow 0 \quad \text { a.s. as } n \rightarrow \infty \text {. }
$$

So we can apply Theorem VII.3 of [8] (with $b_{k}=1 / k$ ) to deduce that

$$
\frac{1}{\left|x^{n}\right|} \sum_{k=1}^{\left\lfloor\left|x^{n}\right| s\right\rfloor}\left(q_{i}(k)-\mathrm{E}\left[q_{i}(k) \mid W^{x^{n}}(k-1)\right]\right) \rightarrow 0 \quad \text { a.s. }
$$

as $n \rightarrow \infty$.

It remains to find the limit of the first term on the right-hand side of (3). Decompose this term as follows:

$$
\frac{1}{\left|x^{n}\right|} \sum_{k=0}^{\left\lfloor\left|x^{n}\right| s\right\rfloor-1}\left(\lambda_{i}-G_{i}\left(W^{x^{n}}(k)\right)\right)=\frac{1}{\left|x^{n}\right|} \sum_{k=0}^{\left\lfloor\left|x^{n}\right| s\right\rfloor-1}\left(\lambda_{i}-\widetilde{G}_{i}\left(X^{n}\left(\frac{k}{\left|x^{n}\right|}\right)\right)\right)+\varepsilon(s, n),
$$

where, by Lemma 3,

$$
|\varepsilon(s, n)| \leq C \frac{1}{\left|x^{n}\right|} \sum_{k=0}^{\left\lfloor\left|x^{n}\right| s\right\rfloor-1} \frac{1}{W_{i}^{x^{n}}(k)} \leq C \frac{1}{\left|x^{n}\right|} \sum_{k=0}^{\left\lfloor\left|x^{n}\right| s\right\rfloor-1} \frac{1}{x_{i}^{n}-k} \rightarrow 0
$$

as $n \rightarrow \infty$ uniformly in $s \leq x_{i}$. Furthermore, from the uniform convergence of $X^{n}$ to $z$ and the continuity of $\widetilde{G}$, we deduce that

$$
\frac{z_{i}(s)-z_{i}(0)}{s}=\lambda_{i}-\lim _{n \rightarrow \infty} \frac{\sum_{k=0}^{\left\lfloor\left|x^{n}\right| s\right\rfloor-1} \widetilde{G}_{i}\left(z\left(k /\left|x^{n}\right|\right)\right)}{\left|x^{n}\right| s} .
$$

Since $\left(1 /\left|x^{n}\right|\right) \sum_{k=1}^{\left\lfloor\left|x^{n}\right| s\right\rfloor} \widetilde{G}_{i}\left(z\left((k-1) /\left|x^{n}\right|\right)\right)$ is a Riemann sum of a continuous bounded function, it converges to $\int_{0}^{s} \widetilde{G}_{i}(z(u)) \mathrm{d} u$, so

$$
\lim _{s \rightarrow 0} \frac{z_{i}(s)-z_{i}(0)}{s}=\lambda_{i}-\lim _{s \rightarrow 0} \frac{\int_{0}^{s} \widetilde{G}_{i}(z(u)) \mathrm{d} u}{s}=\lambda_{i}-\widetilde{G}_{i}(z(0)) .
$$

Case 2: $z_{i}(0)=0$ and $\sum_{j \in V_{i}} z_{j}(0)>0$. Note that $\widetilde{G}_{i}\left(z_{i}(0)\right)=0$. In view of (3) and (4), it suffices to show that

$$
\lim _{s \rightarrow 0^{+}} \lim _{n \rightarrow \infty} \frac{1}{\left|x^{n}\right| s} \sum_{k=0}^{\left\lfloor\left|x^{n}\right| s\right\rfloor-1} G_{i}\left(W^{x^{n}}(k)\right)=0 .
$$

By the assumption, there exists $j \in V_{i}$ such that $z_{j}(0)=\lim _{n \rightarrow \infty} x_{j}^{n} /\left|x^{n}\right|>\alpha>0$. Let $\varepsilon>0$. Then there exists an $n_{0}$ such that, for all $n \geq n_{0}, x_{j}^{n} /\left|x^{n}\right|>\alpha$ and $x_{i}^{n} /\left|x^{n}\right|<\varepsilon$.

Fix $0<s<\alpha$ and $\varepsilon<\alpha$. Then, for large enough $n, W_{i}^{x^{n}}(k) \leq \varepsilon\left|x^{n}\right|+A_{i}(0, k)$, $W_{j}^{x^{n}}(k) \geq \alpha\left|x^{n}\right|-k$, and

$$
G_{i}\left(W^{x^{n}}(k)\right) \leq \frac{W_{i}^{x^{n}}(k)}{W_{i}^{x^{n}}(k)+W_{j}^{x^{n}}(k)} \leq \frac{\varepsilon\left|x^{n}\right|+A_{i}(0, k)}{(\alpha+\varepsilon)\left|x^{n}\right|-k} .
$$


By the strong law of large numbers, $\lim _{t \rightarrow+\infty} A_{i}(0, t) / t=\lambda_{i}$ a.s. Let $\tilde{\lambda}>\lambda_{i}$. We may choose $|x|$ and $n$ large enough and then $k_{0}$ such that, for $k_{0} \leq k \leq s\left|x^{n}\right|$,

$$
G_{i}\left(W^{x^{n}}(k)\right) \leq \frac{\varepsilon\left|x^{n}\right|+\tilde{\lambda} k}{(\alpha+\varepsilon)\left|x^{n}\right|-k}
$$

and

$$
\frac{1}{\left|x^{n}\right|} \sum_{k=0}^{\left\lfloor\left|x^{n}\right| s\right\rfloor-1} G_{i}\left(W^{x^{n}}(k)\right) \leq \frac{k_{0}}{\left|x^{n}\right|}+\frac{\varepsilon}{\alpha+\varepsilon-s}+\frac{1}{\left|x^{n}\right|} \sum_{k=0}^{\left\lfloor\left|x^{n}\right| s\right\rfloor-1} \frac{k \tilde{\lambda}}{\alpha\left|x^{n}\right|-k} .
$$

Direct computations show that

$$
\lim _{n \rightarrow \infty} \frac{1}{\left|x^{n}\right|} \sum_{k=0}^{\left\lfloor\left|x^{n}\right| s\right\rfloor-1} \frac{k \tilde{\lambda}}{\alpha\left|x^{n}\right|-k}=-\tilde{\lambda}\left(s+\alpha \ln \left(1-\frac{s}{\alpha}\right)\right) .
$$

Then

$$
\limsup _{n} \frac{1}{\left|x^{n}\right|} \sum_{k=0}^{\left\lfloor\left|x^{n}\right| s\right\rfloor-1} G_{i}\left(W^{x^{n}}(k)\right) \leq \frac{\varepsilon}{\alpha+\varepsilon-s}-\tilde{\lambda}\left(s+\alpha \ln \left(1-\frac{s}{\alpha}\right)\right) \text { a.s. }
$$

Since the last inequality holds for all $\varepsilon>0$ and $\tilde{\lambda}>\lambda_{i}$, we have

$$
\limsup _{n} \frac{1}{\left|x^{n}\right|} \sum_{k=0}^{\left\lfloor\left|x^{n}\right| s\right\rfloor-1} G_{i}\left(W^{x^{n}}(k)\right) \leq-\lambda_{i}\left(s+\alpha \ln \left(1-\frac{s}{\alpha}\right)\right) .
$$

It then follows immediately that

$$
\lim _{s \rightarrow 0^{+}} \limsup _{n} \frac{1}{\left|x^{n}\right| s} \sum_{k=0}^{\left\lfloor\left|x^{n}\right| s\right\rfloor-1} G_{i}\left(W^{x^{n}}(k)\right)=0 .
$$

The proof of Theorem 3 is now complete.

We also need a further result that may be deduced from Theorem 3 . Let $H=\left\{x \in \mathbb{R}^{K}: x_{i}>\right.$ 0 for all $i=1, \ldots, K\}$ be the interior of the positive orthant.

Lemma 4. Assume that $z(0) \in H$. Then either

(i) there exists a c such that $z(c)=0$ and $z(t) \in H$ for all $t \in(0, c)$, or

(ii) $z(t)$ remains in $H$ for all $t>0$.

Proof. Restricted on the open set $H$, the right-hand side of (2) is a continuous function. Therefore, the solutions of (2) are locally uniquely defined as long as $z(t)$ remains in $H$. Now, suppose, to the contrary, that $t \mapsto z(t)$ leaves $H$ at time $c$ at a point $y=\lim _{t \rightarrow c-} z(t) \in \partial H \backslash\{0\}$.

Let $a_{i}=\limsup _{t \rightarrow c^{-}} \phi(z(t)), a_{i} \in[0,1]$. Since $y \neq 0$, there exist $i_{1}$ and $i_{2}$ such that $y_{i_{1}}=0$ and $y_{i_{2}}>0$. The connectivity of $G$ implies that there exists a $k$ such that $y_{k}=0$ and $\sum_{j \in V_{k}} y_{k}>0$ (for this, consider any path from $i_{1}$ to $i_{2}$ ). Hence, $a_{k}=0$ and $\lim _{t \rightarrow c^{-}} F_{k}(\phi(z(t)))=\lambda>0$; this implies that $t \mapsto x_{k}(t)$ increases in a left neighbourhood of $c$, which contradicts $y_{k}=\lim _{t \rightarrow c-} z(t)=0$.

Lemma 4 implies that, for an initial condition in $H$, the fluid limit $z(t)$ remains in $H$ or reaches 0 at time $c$. It also implies that if $z(0)=\lim _{n} x^{n} /\left|x^{n}\right| \in H$ then the fluid limit is deterministic. 


\section{Properties of the fluid limit on the boundary}

In this section we work with the general case (the symmetry assumptions are no longer applied in this case).

Conjecture 1. We conjecture that all coordinates of any fluid limit $z$ have right derivatives at point 0 (even if there exists an $i$ such that $x_{j}=z_{j}(0)=0$ for all $j \in V_{i}$ ). We also conjecture that the right derivative, $z^{\prime}(0)$, of a fluid limit $\{z(t), t \geq 0\}$ does not depend on the sequence $x^{n}$ and depends only on $x=\lim _{n} x^{n} /\left|x^{n}\right|$. If this is true then all fluid limits are deterministic functions.

In this section we prove a weaker statement which implies that the boundary of the positive orthant does not play any role in determining the stability conditions for the fluid model. Define

$$
\tau_{h}=\inf \{t \geq 0:|z(t)|<h\} .
$$

Also, define $\lambda_{*}=\min \left\{\lambda_{1}, \ldots, \lambda_{K}\right\}>0$. Since $|z(0)|=1, \max _{i} z_{i}(0) \geq 1 / K$. The inequality $z_{i}^{\prime}(t) \geq \lambda_{*}-1$ for all $i$ and $t$ also implies that

$$
\tau_{1-\varepsilon} \geq \frac{\varepsilon}{K\left(1-\lambda_{*}\right)}
$$

Theorem 4. There exist positive constants $b$ and $\varepsilon_{0}$ such that, for any $\varepsilon \in\left(0, \varepsilon_{0}\right), \min _{i} z_{i}(t) \geq$ $b \varepsilon$ for any $t \in\left[c \varepsilon, \tau_{1-\varepsilon}\right)$, where $c=1 / K\left(1-\lambda_{*}\right)$.

From the self-similar property of fluid limits, the following corollary is immediate.

Corollary 2. For any $h>0, z_{i}(t)>0$ for all $0<t<\tau_{h}$ and all $i$.

Lemma 4 and Corollary 2 imply the following.

Corollary 3. Assume that $|z(0)|>0$. Then either

(i) there exists a c such that $z(c)=0$ and $z(t)$ remains in $H$ for all $t \in(0, c)$, or

(ii) $z(t)$ remains in $H$ for all $t>0$.

We do not present proofs of these statements here as they are rather obvious.

The rest of this section is devoted to the proof of Theorem 4. We begin with two technical lemmas.

Lemma 5. There exist positive constants $K_{1}>1$ and $K_{2}$ such that, for any fluid limit $z$, if $z_{i}(t)>K_{1} z_{j}(t)$ for two neighbouring nodes $i$ and $j$, then $z_{j}^{\prime}(t)>K_{2}$.

Proof. The existence of $z_{j}^{\prime}(t)$ is guaranteed by Theorem 3. Since $\sum_{k \in V_{j}} z_{k}(t)>z_{i}(t)>0$, we have

$$
z_{j}^{\prime}(t)>\lambda_{*}-\frac{z_{j}(t)}{\sum_{k \in V_{j}} z_{k}(t)} \geq \lambda_{*}-\frac{z_{j}(t)}{z_{i}(t)+z_{j}(t)}>\lambda_{*}-\frac{1}{1+K_{1}},
$$

and we may take $K_{1}=2 / \lambda_{*}-1$ and $K_{2}=\lambda_{*} / 2$.

Lemma 6. There exist constants $C_{1} \geq C_{2}>0$ such that, for any $h>0$, we can choose $h_{1}>0$ such that if $|z(0)| \geq h_{1}$ and $\min _{i} z_{i}(0) \geq C_{1}$ h then $\min _{i} z_{i}(t) \geq C_{2} h_{1}$ for all $t \leq \tau_{h}$. 
Proof. Denote by $D$ the maximum graph distance in $g$ (the diameter of $g$ ). Set $C_{1}=$ $1 / K K_{1}^{D+1}$ and $C_{2}=C_{1} / K_{1}^{D-1}$. We prove Lemma 6 for $h=1$. The result for an arbitrary $h$ follows from the self-similarity of fluid limits.

It is sufficient to show that, for any $t<\tau_{1}$, if $\min _{i} z_{i}(t) \geq C_{1}$ then there exists $0<s<\infty$ such that

$$
\min _{i} z_{i}(t+s) \geq C_{1}
$$

and

$$
\min _{i} z_{i}(u) \geq C_{2} \quad \text { for all } t \leq u \leq t+s
$$

Indeed, assume that (5)-(6) hold and that Lemma 6 is not valid. Then there exists a $t \leq \tau_{1}$ such that $\min _{i} z_{i}(t)<C_{2}$. It then follows from the continuity of fluid limits that there is a last moment $v<t$ when $\min _{i} z_{i}(v) \geq C_{1}$. However, it follows from (5)-(6) that there exists an $s>0$ such that $\min _{i} z_{i}(v+s) \geq C_{1}$ and $\min _{i} z_{i}(u) \geq C_{2}$ for all $v \leq u \leq v+s$. Clearly, $v+s<t$, which contradicts our assumption that $v$ is a last moment before $t$ when $\min _{i} z_{i}(v) \geq C_{1}$.

Now let $t$ be any time such that $t<\tau_{1}$ and $\min _{i} z_{i}(t) \geq C_{1}$. Note that $\max _{i} z_{i}(t) \geq 1 / N=$ $C_{1} K_{1}^{D+1}$ since $t<\tau_{1}$. To simplify the notation, assume that $z_{1}(t)=\max _{i} z_{i}(t)$. Let $T$ be such that $z_{1}(t+u) \geq C_{1} K_{1}^{D}$ for all $0 \leq u \leq T$. Again, note that $z_{i}^{\prime}(u) \geq \lambda_{*}-1$ for all $i$ and $u$. This implies that

$$
T \geq \frac{C_{1}\left(K_{1}^{D+1}-K_{1}^{D}\right)}{1-\lambda_{*}}=\frac{C_{1} K_{1}^{D}\left(K_{1}-1\right)}{1-\lambda_{*}} .
$$

Let $d$ be the maximum distance in $g$ from node 1 . Clearly, $d \leq D$. For $j=1, \ldots, d$, denote by $A_{j}$ the set of nodes at distance $j$ from node 1 .

We show that there exists $0<s<T$ such that (5) and (6) hold. First, we show that $\min z_{i}(u) \geq C_{2}$ for all $t \leq u \leq t+T$. Note that $z_{i}(u) \geq C_{1}$ for all $i \in A_{1}$ and $t \leq u<t+T$. Indeed, assume that there exist $i \in A_{1}$ and $t \leq u<t+T$ such that $z_{i}(u)<C_{1}$. Then, by continuity, there is a last moment $t \leq u_{1}<u$ such that $z_{i}\left(u_{1}\right) \geq C_{1}$. Lemma 5 implies that $z_{i}^{\prime}\left(u_{1}\right) \geq K_{2}>0$ and, hence, there exists a time $u_{2}>u_{1}$ such that $z_{i}\left(u_{2}\right) \geq C_{1}$; but this contradicts our assumption on $u_{1}$. Using induction and following the same arguments as above, we can show that $z_{i}(u) \geq C_{1} / K_{1}^{j-1}$ for all $i \in A_{j}$ and $t \leq u \leq t+T$ for any $j=1, \ldots, d$. Hence, $\min _{i} z_{i}(u) \geq C_{1} / K_{1}^{d-1} \geq C_{1} / K_{1}^{D-1}=C_{2}$ for all $\bar{t} \leq \bar{u} \leq t+T$.

Let us now show that there exists $0<s<T$ such that (5) holds. For every $j=1, \ldots, d$, denote by $t_{j}$ the time needed to achieve the level $C_{1} K_{1}^{d-j}$ starting from the level $C_{1} / K_{1}^{j-1}$ and moving with speed $K_{2}$. Clearly, $t_{j}=C_{1}\left(K_{1}^{d-1}-1\right) / K_{2} K_{1}^{j-1}$. Note that (5) and (6) hold with $s=\sum_{j=1}^{d} t_{j}$ if $T \geq \sum_{j=1}^{d} t_{j}$. Indeed, $\min _{j \in A_{1}} z_{j}$ will achieve the level $C_{1} K_{1}^{d-1}$ not later than time $t+t_{1}$ and it will not become smaller than this level before time $t+T$, since all nodes in $A_{1}$ are neighbours of node 1 and $z_{1}(u) \geq K_{1}^{D}$ for all $t \leq u \leq t+T$. Note also that $\min _{j \in A_{2}} z_{j}$ will become greater than $C_{1} K_{1}^{d-2}$ not later than time $t+t_{1}+t_{2}$ since it cannot become smaller than $C_{1} / K_{1}$ before time $t+t_{1}$, and after this time it is either greater than $C_{1} K_{1}^{d-2}$ or grows with a speed of at least $K_{2}$ (this follows from Lemma 5 and the fact that any node in $A_{2}$ has a neighbour in $A_{1}$ ). We can continue these arguments to prove that $\min _{j \in A_{d}} z_{j}$ will become greater than $C_{1}$ not later than time $t+\sum_{i=1}^{d} t_{i}$ if $T \geq \sum_{i=1}^{d} t_{i}$. 
Note that

$$
\begin{aligned}
\sum_{i=1}^{d} t_{i} & =\frac{C_{1}\left(K_{1}^{d-1}-1\right)\left(1+K_{1}+\cdots+K_{1}^{d-1}\right)}{K_{2} K_{1}^{d-1}}=\frac{C_{1}\left(K_{1}^{d-1}-1\right)\left(K_{1}^{d}-1\right)}{K_{2} K_{1}^{d-1}\left(K_{1}-1\right)} \\
& \leq \frac{C_{1}\left(K_{1}^{d}-1\right)}{K_{2}\left(K_{1}-1\right)} \\
& \leq \frac{C_{1}\left(K_{1}^{D}-1\right)}{K_{2}\left(K_{1}-1\right)} .
\end{aligned}
$$

If we take $K_{2}=\lambda_{*} / 2$ and $K_{1}=2 / \lambda_{*}-1$, then $\left(1-\lambda_{*}\right) / K_{2}=K_{1}-1$. Note also that in this case $K_{1} \geq 2$. It now follows from (7) and (8) that $T \geq \sum_{i=1}^{d} t_{i}$.

We can see from the proof of Lemma 6 that the following (stronger) result holds.

Lemma 7. For any $h_{1}>0$, there exists an $\hat{h}_{2}>0$ such that, for any $h_{2} \leq \hat{h}_{2}$, there exists $0<h_{3} \leq h_{2}$ such that if $|z(0)| \geq h_{1}$ and $\min _{i} z_{i}(0) \geq h_{2}$ then $\min _{i} z_{i}(t) \geq h_{3}$ for all $t \leq \tau_{h_{1}}$.

Remark 1. Lemma 7 is valid with $\hat{h}_{2}=h_{1} / K K_{1}^{D+1}$.

Proof of Theorem 4. The proof of Theorem 4 is similar to that of Lemma 6. Recall that $D$ is the maximum graph distance of $g_{\text {. Take }} \varepsilon_{0}$ such that $K_{2}\left(K_{1}-1\right) \varepsilon /\left(K_{1}^{D}-1\right) \leq(1-\varepsilon) / K K_{1}^{D+1}$ for all $\varepsilon \leq \varepsilon_{0}$, and let $a=K_{2}\left(K_{1}-1\right) /\left(K_{1}^{D}-1\right)$. Then $a \varepsilon \leq(1-\varepsilon) / K K_{1}^{D+1}$, and, in view of Lemma 7 and Remark 1, it is enough to prove that $\min _{i} z_{i}(c \varepsilon) \geq a \varepsilon$.

Note that $\max _{i} z_{i}(0) \geq 1 / K$. Assume that $z_{1}(0)=\max _{i} z_{i}(0)$. Let $T$ be such that $z_{1}(u) \geq$ $a \varepsilon K_{1}^{D}$ for all $0 \leq u \leq T$. Then $z_{i}^{\prime}(t) \geq \lambda_{*}-1$ implies that

$$
T \geq \frac{1 / K-a \varepsilon K_{1}^{D}}{1-\lambda_{*}}=\frac{1-K a \varepsilon K_{1}^{D}}{K\left(1-\lambda_{*}\right)} .
$$

Again, let $d$ be the maximum distance in $g$ from node 1 . For $j=1, \ldots, d$, denote by $A_{j}$ the set of nodes at distance $j$ from node 1 . For every $j=1, \ldots, d$, denote by $t_{j}$ the time needed to achieve the level $a \varepsilon K_{1}^{d-j}$ starting from the level 0 and moving with speed $K_{2}$. Clearly, $t_{j}=a \varepsilon K_{1}^{d-j} / K_{2}$. Define $T_{1}=\sum_{j=1}^{D} t_{j}$. Note that

$$
T_{1}=\frac{a \varepsilon\left(K_{1}^{D}-1\right)}{K_{2}\left(K_{1}-1\right)}=\frac{\varepsilon}{K\left(1-\lambda_{*}\right)}=c \varepsilon .
$$

Following the same arguments as in the proof of Lemma 6 , we can show that $\min _{i} z_{i}(c \varepsilon)=$ $\min _{i} z_{i}\left(T_{1}\right) \geq a \varepsilon$ if $T_{1} \leq T$.

It remains to prove that $T_{1} \leq T$. This follows from (9), (10), and the inequality $a \varepsilon \leq$ $(1-\varepsilon) / K K_{1}^{D+1}$.

Remark 2. Denote by $v(s, h, b)=\inf \left\{t \geq s:|z(t)|<h\right.$ or $\left.\min _{i} z_{i}(t)<b\right\}$ the time (after moment $s$ ) of the first exit from the set $\{|z| \geq h\} \cap\left\{\min _{i} z_{i} \geq b\right\}$ after time $s$. Theorem 4 implies that there exist $b>0$ and $s \geq 0$ such that $\tau_{1-\varepsilon}=v(s \varepsilon, 1-\varepsilon, b \varepsilon)$ for any initial condition $z(0)$.

\section{Proof of Theorem 2}

In this section we first present a proof of our main stability result and then formulate its generalisation. Recall that Theorem 1 follows from Theorem 2 and Lemma 2.

Recall also that here we deal with the symmetric case. We need to prove that there exists a deterministic time $t_{0}$ such that, for all fluid limits, $z(t)=0$ for $t \geq t_{0}$ a.s. 
Lemma 8. If $z_{i}(t)>0$ for all $i=1, \ldots, K$ then

$$
\left(\sum_{i} z_{i}^{2}(t)\right)^{\prime} \leq\left(\lambda-\frac{\mathrm{e}^{-1}}{V}\right) \sum_{i} z_{i}(t)
$$

and, hence, if $\lambda<\mathrm{e}^{-1} / V$,

$$
\left(\sum_{i} z_{i}^{2}(t)\right)^{\prime} \leq-\varepsilon \sum_{i} z_{i}(t) \text { for some } \varepsilon>0 .
$$

Proof. Clearly, it is sufficient to prove the inequality

$$
\frac{\sum_{i} z_{i} \varphi_{i} \exp \left\{-\sum_{j \in V_{i}} \varphi_{j}\right\}}{\sum_{k} z_{k}} \geq \frac{\mathrm{e}^{-1}}{V},
$$

where we have slightly abused the notation used in (1) by writing $z_{i}$ instead of $z_{i}(t)$ and $\varphi_{i}$ instead of $\varphi_{i}(t)$. We can write the left-hand side of (11) in the form

$$
\sum_{i} p_{i} f\left(y_{i}\right)
$$

where $p_{i}=z_{i} / \sum_{k} z_{k}, y_{i}=-\sum_{j \in V_{i}} \varphi_{j}-\ln \left(1 / \varphi_{i}\right)$, and $f(z)=\mathrm{e}^{z}$. Function $f$ is convex and $\sum_{i} p_{i}=1$; hence, $\sum_{i} p_{i} f\left(y_{i}\right) \geq f\left(\sum_{i} p_{i} y_{i}\right)$ and

$$
\frac{\sum_{i} z_{i} \varphi_{i} \exp \left\{-\sum_{j \in V_{i}} \varphi_{j}\right\}}{\sum_{k} z_{k}} \geq \exp \left\{-\sum_{i} \frac{z_{i}}{\sum_{k} z_{k}} \sum_{j \in V_{i}} \varphi_{j}-\sum_{i} \frac{z_{i}}{\sum_{k} z_{k}} \ln \frac{1}{\varphi_{i}}\right\} \text {. }
$$

Now consider

$$
\sum_{i} \frac{z_{i}}{\sum_{k} z_{k}} \sum_{j \in V_{i}} \varphi_{j} \quad \text { and } \quad \sum_{i} \frac{z_{i}}{\sum_{k} z_{k}} \ln \frac{1}{\varphi_{i}}
$$

separately. We have

$$
\sum_{i} \frac{z_{i}}{\sum_{k} z_{k}} \sum_{j \in V_{i}} \varphi_{j}=\frac{\sum_{i} z_{i} \sum_{j \in V_{i}} \varphi_{j}}{\sum_{k} z_{k}}=\frac{\sum_{j} \varphi_{j} \sum_{i \in V_{j}} z_{i}}{\sum_{k} z_{k}}=\frac{\sum_{j} z_{j}}{\sum_{k} z_{k}}=1
$$

(where we have used the identity $\varphi_{j} \sum_{i \in V_{j}} z_{i}=z_{j}$ and the symmetry of the neighbourhood relation: $j \in V_{i}$ if and only if $i \in V_{j}$ ).

Note that the logarithmic function is concave. Hence

$$
\sum_{i} \frac{z_{i}}{\sum_{k} z_{k}} \ln \frac{1}{\varphi_{i}} \leq \ln \left(\sum_{i} \frac{z_{i}}{\sum_{k} z_{k}} \frac{1}{\varphi_{i}}\right)=\ln \left(\frac{\sum_{i} z_{i} / \varphi_{i}}{\sum_{k} z_{k}}\right)=\ln \left(\frac{\sum_{i} \sum_{j \in V_{i}} z_{j}}{\sum_{k} z_{k}}\right)=\ln V .
$$

Inequality (11) now follows from (12), (13), and (14).

Proof of Theorem 2. Corollary 2 implies that $z_{i}(t)>0$ for all $i=1, \ldots, K$ and all $0<$ $t<\inf \{u: z(u)=0\}$. Then we can use Lemma 8. Note also that $\sum_{i} x_{i} \geq \sqrt{\sum_{i} x_{i}^{2}}$ for any positive values of $\left\{x_{i}\right\}$. Hence, Lemma 8 implies that

$$
\left(\sum_{i} z_{i}^{2}(t)\right)^{\prime} \leq-\varepsilon \sqrt{\sum_{i} z_{i}^{2}(t)}
$$


Then

$$
\left(\sqrt{\sum_{i} z_{i}^{2}(t)}\right)^{\prime} \leq-\frac{\varepsilon}{2}
$$

and the result follows.

Remark 3. By applying the methods of [18], we can obtain a similar (but less explicit) stability result in a more general situation. Assume now that the system may be asymmetric, i.e. that the values of $\lambda_{i}$ may differ for different $i$ and the graph $g$ may be irregular.

Let

$$
M=\left\{\mu: \mu_{i}=p_{i} \exp \left\{-\sum_{j \in V_{i}} p_{j}\right\}, i=1, \ldots, K, \text { for some } \bar{p}=\left(p_{1}, \ldots, p_{K}\right), p_{i} \geq 0\right\}
$$

We can show that the vector $\left(\varphi_{1}, \ldots, \varphi_{K}\right)$ with $\varphi_{i}=z_{i} / \sum_{j \in V_{i}} z_{j}$ maximises the function $\sum_{i=1}^{K} z_{i} \ln \mu_{i}$ over all vectors $\mu \in M$. Based on this, we can obtain the following.

Theorem 5. Assume that there exists a vector $\mu \in M$ such that $\lambda<\mu$ componentwise. Then the Markov chain $W_{n}$ is positive recurrent.

A proof of Theorem 5 follows the lines of the proof of Theorem 4 of [18].

\section{Rate of convergence}

In this section we again consider the symmetric case. We will obtain power rates of convergence of $W_{n}$ to its stationary distribution in the total variation norm. We expect that we can similarly prove the geometric ergodicity of the underlying Markov chain given the light-tailedness of the distribution of the increments $\{A(n)\}$.

Define the total variation distance between distributions $\pi_{1}$ and $\pi_{2}$ by

$$
\left\|\pi_{1}(\cdot)-\pi_{2}(\cdot)\right\|=\sup _{|g| \leq 1}\left|\int g(y) \pi_{1}(\mathrm{~d} y)-\int g(y) \pi_{2}(\mathrm{~d} y)\right| .
$$

Theorem 6. Assume that $\lambda<\mathrm{e}^{-1} / V$ and that $\mathrm{E} A_{i}(n)^{p+1}<\infty$ for some $p \geq 1$ and all $i=1, \ldots, K$ and $n$. Assume also that $\mathrm{P}\left(A_{1}(1)=0, A_{2}(1)=0, \ldots, A_{K}(1)=0\right)>0$. Then

$$
\lim _{n \rightarrow \infty} n^{p}\left\|\mathrm{P}^{n}(x, \cdot)-\pi(\cdot)\right\|=0, \quad x \in \mathbb{N}^{K},
$$

where $\mathrm{P}^{n}(x, \cdot)$ is the distribution of $W^{x}(n)$ and $\pi(\cdot)$ is the stationary distribution of $W$.

Proof. The proof of Theorem 6 is based on the following lemma, which is an analogue of Proposition 5.3 of [4].

Lemma 9. Assume that the conditions of Theorem 6 are satisfied. Then, for some constants $c<\infty$ and $\delta>0$, and a finite set $C$,

$$
\mathrm{E}\left[\sum_{n=0}^{\tau_{C}(\delta)}\left|W^{x}(n)\right|^{p}\right] \leq c|x|^{p+1} \text { for any } x \in \mathbb{N}^{K},
$$

where $\tau_{C}(\delta)=\min \{n \geq \delta: W(n) \in C\}$. 
Proof. The proof of Lemma 9 follows the lines of the proof of Proposition 5.3 of [4]. It follows from Theorem 2 that there exists a $t_{0}$ such that

$$
\lim _{|x| \rightarrow \infty} \frac{W^{x}\left(|x| t_{0}\right)}{|x|}=0 \quad \text { a.s. }
$$

Note also that the family of random variables $\left\{\left|W^{x}\left(|x| t_{0}\right)\right|^{p+1} /|x|^{p+1}\right\}$ is uniformly integrable, since

$$
\frac{\left|W^{x}\left(|x| t_{0}\right)\right|^{p+1}}{|x|^{p+1}} \leq \frac{\left(\sum_{m=0}^{|x| t_{0}} \sum_{i=1}^{K} A_{i}(m)\right)^{p+1}}{|x|^{p+1}} \leq t_{0}^{p+1} \frac{\sum_{m=0}^{|x| t_{0}}\left(\sum_{i=1}^{K} A_{i}(m)\right)^{p+1}}{|x| t_{0}}
$$

and the family $\left\{\sum_{m=0}^{|x| t_{0}}\left(\sum_{i=1}^{K} A_{i}(m)\right)^{p+1} /|x| t_{0}\right\}$ is uniformly integrable. This is guaranteed by the existence of $\mathrm{E} A_{i}(m)^{p+1}$ for all $i=1, \ldots, K$ and all $m$. Hence,

$$
\lim _{|x| \rightarrow \infty} \frac{\mathrm{E}\left[\left|W^{x}\left(|x| t_{0}\right)\right|^{p+1}\right]}{|x|^{p+1}}=0 .
$$

Choose $L$ such that

$$
\mathrm{E}\left[\left|W^{x}\left(|x| t_{0}\right)\right|^{p+1}\right] \leq \frac{1}{2}|x|^{p+1} \text { for }|x| \geq L .
$$

Define, as in the proof of Proposition 5.3 of [4], the sequence of stopping times $\sigma_{0}=0, \sigma_{1}=$ $t(x)$, and $\sigma_{k+1}=\sigma_{k}+\theta_{\sigma_{k}} \sigma_{1}, k \geq 1$, where $t(x)=t_{0} \max \{L,|x|\}$ and $\theta$ is the shift operator on the sample space. We assume that $t_{0}$ is an integer. The stochastic process $\hat{W}_{k}=W\left(\sigma_{k}\right)$ is a Markov chain with transition kernel

$$
\hat{P}(x, A)=\mathrm{P}\left(W^{x}(t(x)) \in A\right) .
$$

Now (15) implies that

$$
\mathrm{E}\left[\left|\hat{W}_{1}\right|^{p+1}-\left|\hat{W}_{0}\right|^{p+1} \mid \hat{W}_{0}=x\right] \leq-\frac{1}{2}|x|^{p+1}+b I_{C}(x)
$$

for the set $C=\{x:|x| \leq L\}$ and some constant $b$. The comparison theorem (see [13, p. 337]) yields

$$
\mathrm{E}\left[\sum_{n=0}^{k_{*}-1}\left|W^{x}\left(\sigma_{k}\right)\right|^{p+1}\right]=\mathrm{E}\left[\sum_{n=0}^{k_{*}-1}|\hat{W}(k)|^{p+1}\right] \leq 2\left(|x|^{p+1}+b I_{C}(x)\right)
$$

where $k_{*}=\min \{k \geq 1: \hat{W}(k) \in C\}$. To prove Lemma 9, we first show that, for some constant $c_{0}$,

$$
\mathrm{E}\left[\sum_{n=\sigma_{k}}^{\sigma_{k+1}}\left|W^{x}(n)\right|^{p} \mid \mathcal{F}_{\sigma_{k}}\right] \leq c_{0} W^{x}\left(\sigma_{k}\right)^{p+1}
$$

which, by the strong Markov property, amounts to

$$
\mathrm{E} \sum_{n=0}^{t(x)}\left|W^{x}(n)\right|^{p} \leq c_{0}|x|^{p+1}
$$

This follows from the fact that

$$
\sum_{n=0}^{t(x)}\left|W^{x}(n)\right|^{p} \leq \sum_{n=0}^{t(x)}\left(\sum_{m=0}^{n} \sum_{i=1}^{K} A_{i}(m)\right)^{p} \leq \sum_{n=0}^{t(x)}\left(\sum_{m=0}^{t(x)} \sum_{i=1}^{K} A_{i}(m)\right)^{p} \quad \text { a.s. }
$$


and from our assumption that $\mathrm{E} A_{i}(m)<\infty$ for all $i=1, \ldots, K$ and all $m$. Substituting (17) into (16), we have

$$
\mathrm{E}\left[\sum_{k=0}^{\infty} \mathrm{E}\left[\sum_{n=\sigma_{k}}^{\sigma_{k+1}}\left|W^{x}(n)\right|^{p} \mid \mathcal{F}_{\sigma_{k}}\right] \mathbf{I}\left\{k<k_{*}\right\}\right] \leq c|x|^{p+1} .
$$

By Fubini's theorem and the smoothing property of the conditional expectation, the left-hand side is precisely $\mathrm{E}\left[\sum_{n=0}^{\sigma_{k_{*}}}\left(1+\left|W^{x}(n)\right|^{p}\right)\right]$. The lemma now follows from the fact that $\sigma_{k_{*}} \geq$ $\tau_{C}\left(t_{0} L\right)$.

We now apply Proposition 5.4 of [4] with $t=1$. In our case, it gives the following bound:

$$
\mathrm{E}[V(W(1))-V(W(0)) \mid W(0)=x] \leq-f(x)+\kappa
$$

with $V(x)=\mathrm{E}\left[\sum_{n=0}^{\tau_{C}(\delta)}\left|W^{x}(n)\right|^{p}\right]$ and $f(x)=|x|^{p}$.

Furthermore, Lemma 9 implies that $V(x) \leq c|x|^{p+1}$. Now (18) yields

$$
\mathrm{E}[V(W(1))-V(W(0)) \mid W(0)=x] \leq V(x)^{p /(p+1)}+b I_{C}
$$

for the set $C=\{x:|x| \leq L\}$ and some constant $b$. Theorem 6 now follows from Theorem 2.5 of [5].

\section{Local stability of fluid limits on the positive orthant}

In this section we investigate the behaviour of the solution to differential equation (2). In particular, we show that if the input rate $\lambda$ is sufficiently small then the diagonal is locally unstable.

\subsection{Orbits of the fluid limits}

Recall that $H=\left\{x \in \mathbb{R}^{K}: x_{i}>0\right.$ for all $\left.i=1, \ldots, K\right\}$ is the interior of the positive orthant and that $\mathbf{1}=(1, \ldots, 1)$. For $z(t)$ in $H$, differential equation (2) may be restated in a closed form as

$$
z^{\prime}(t)=F(\phi(z(t)))
$$

with $F_{i}(x)=\lambda-x_{i} \exp \left\{-\sum_{j \in V_{i}} x_{j}\right\}$. Let $\Delta=\left\{x \in H: x_{1}=x_{2}=\cdots=x_{K}\right\}$ be the main diagonal, and let $C_{u}=\{x \in H:|x /| x|-\mathbf{1} / K| \leq u\}, u>0$, be a cone with direction $\Delta$. We note that the main diagonal is an orbit of the equation $F(\phi(c \mathbf{1}))=\left(\lambda-\mathrm{e}^{-1 / V)} \mathbf{1}\right.$.

Let $A$ be the adjacency matrix of $g$ and let $\left\{v_{1}, \ldots, v_{K}\right\}$ be its eigenvalues with $v_{i} \leq v_{i+1}$. The spectral gap $\gamma$ is defined by

$$
\gamma=\min _{i<K}\left(v_{K}-v_{i}\right)=v_{K}-v_{K-1}
$$

Note that since $g$ is $(V-1)$-regular, $v_{K}=V$. The main result of this section is the following.

Theorem 7. If $\lambda>\left(\mathrm{e}^{-1} / V\right)\left(1-\gamma^{2} / V^{2}\right)$ then there exists a $u>0$ such that, for all solutions $t \rightarrow z(t)$ of (19) with initial condition in $C_{u}$,

$$
\lim _{t \rightarrow+\infty} \phi(z(t))=\frac{1}{V}
$$

If $\lambda<\left(\mathrm{e}^{-1} / V\right)\left(1-\gamma^{2} / V^{2}\right)$ then the diagonal is locally unstable. 
Theorem 7 will be proved in the next subsection.

Corollary 4. Assume that $\lambda>\left(\mathrm{e}^{-1} / V\right)\left(1-\gamma^{2} / V^{2}\right)$ and that $z(t)$ is a solution of (19). There exists a $u>0$ such that if $z(0) \in C_{u}$ then the following statements hold.

(i) If $\lambda<\mathrm{e}^{-1} / V$ then $z(c)=0$ for some $c>0$.

(ii) If $\lambda>\mathrm{e}^{-1} / V$ then $z(t) \sim\left(\lambda-\mathrm{e}^{-1} / V\right) t$.

Proof. Let $z(t)$ be the maximal solution with given initial value $z(0) \in H$. From Theorem 7 , $\lim \phi(z(t))=\mathbf{1} / V$. Since $F$ is continuous in a neighbourhood of $1 / V, \lim _{t \rightarrow+\infty} z^{\prime}(t)=$ $\left(\lambda-\mathrm{e}^{-1} / V\right)$ 1. If $\lambda \neq \mathrm{e}^{-1} / V$ then the latter implies that, as $t$ tends to $\infty$,

$$
z(t) \sim\left(\lambda-\frac{\mathrm{e}^{-1}}{V}\right) t \mathbf{1}
$$

Then the second statement of the corollary follows. Suppose now that $\lambda-\mathrm{e}^{-1} / V<0$. Then, from (20), $z(t)$ leaves $H$ in finite time. Lemma 4 implies in turn that there exists a $c>0$ such that $z(c)=0$. So, the first assertion of Corollary 4 is also proved.

\subsection{Proof of Theorem 7}

The proof of Theorem 7 is an application of the stability theory of differential equations. It will be given in the series of technical lemmas.

6.2.1. Spectral analysis. We need to consider the eigenvalues of $D(F \circ \phi)(x)$ for $x \in \Delta$, where $D f(x)$ is the differential of $f$ at $x$. Here $F \circ \phi$ is homogeneous of order 0 : for all $c>0$, $F(\phi(c x))=F(\phi(x))$. Hence,

$$
D(F \circ \phi)(c \mathbf{1})=c^{-1} D(F \circ \phi)(\mathbf{1}) .
$$

Since $\Delta$ is an orbit of (19), $\mathbf{1}$ is an eigenvector of $D(F \circ \phi)(\mathbf{1})$ associated with the eigenvalue 0 .

Lemma 10. The eigenvalues of $D(F \circ \phi)(\mathbf{1})$ are $\left(0, \eta_{1}, \ldots, \eta_{K-1}\right)$ with $\eta_{i}=-\left(\mathrm{e}^{-1} / V^{3}\right)(V-$ $\left.v_{K-i}\right)^{2}$. In particular, $\eta_{i}<0$ for all $i \geq 1$.

Proof. A direct computation leads to

$$
(D(F \circ \phi)(\mathbf{1}))_{i j}= \begin{cases}-\frac{\mathrm{e}^{-1}(V-1)}{V^{2}} & \text { if } j=i, \\ \frac{\mathrm{e}^{-1}\left|V_{i} \cup V_{j}\right|}{V^{3}} & \text { if } j \in V_{i} \backslash\{i\}, \\ -\frac{\mathrm{e}^{-1}}{V^{3}}\left|V_{i} \cap V_{j}\right| & \text { if } j \notin V_{i} .\end{cases}
$$

Then $D(F \circ \phi)(\mathbf{1}) \cdot \mathbf{1}=0$. To show this, let $M=-\mathrm{e} V^{3} D(F \circ \phi)(\mathbf{1})$. Using the equality $\left|V_{i} \cup V_{j}\right|=2 V-\left|V_{i} \cap V_{j}\right|$, we deduce that

$$
(M \mathbf{1})_{i}=V(V-1)-2 V(V-1)+\sum_{j \neq i}\left|V_{i} \cap V_{j}\right|=\sum_{j=1}^{K}\left|V_{i} \cap V_{j}\right|-V^{2}=0 .
$$


Let $E$ denote the identity matrix, and let $A$ be the adjacency matrix of $g$. Since $\left(A^{2}\right)_{i j}=$ $\left|V_{i} \cap V_{j}\right|$, we have the following decomposition:

$$
M=V^{2} E-2 V A+A^{2}=(A-V E)^{2} .
$$

The matrix $A$ is irreducible since $g$ is connected. Thus, $(A-V E)$ is an ML-matrix (see [16, pp. 45-53]). In graph theory, this matrix is called the Laplacian matrix of $g$. From Corollary 1 of Theorem 1 of [16], the spectral radius of $A$ is $V$. Theorem 2.6(d) of [16] implies that $\operatorname{dim} \operatorname{Ker}(A-V E)=1$ and that all the nonzero eigenvalues of $(A-E)^{2}$ are positive reals (recall that the spectrum of $A$ is real).

6.2.2. Orbit of $\psi \circ z$. We define

$$
\Sigma=\left\{x \in H: \sum_{i=1}^{K} x_{i}=1\right\}=H \cap\langle\mathbf{1}, \cdot\rangle^{-1}(\{1\})=\psi(H),
$$

where $\psi(x)=x /|x|$. Clearly, $\Sigma$ is a $C^{\infty}$-convex manifold of codimension 1 . Introduce the following differential equation on $\Sigma$ :

$$
y^{\prime}=D \psi(y) F(\phi(y))=\alpha(y)
$$

with an initial condition $y(0)$ in $\Sigma$. Here $\alpha$ is a $C^{\infty}(\Sigma)$ function and $\alpha(y) \in T_{y}(\Sigma)$ is the tangent space of $\Sigma$ at $y$. The next step is to compare the orbits of (19) and (21). The next lemma asserts that the orbits of the solution of the equation $y^{\prime}=\alpha(y)$ and $\psi \circ x$ are indeed equal (here $t \mapsto z(t)$ is a solution of (19)).

Lemma 11. Let $z(0)$ belong to $H$, and let $z(t)$ be the maximal solution of (19). Let $y(t)$ be the maximal solution of $y^{\prime}=G(y)$, with initial condition $y(0)=\psi(z(0))$. Then $y(t)$ is defined on $\mathbb{R}_{+}$, and there exists an increasing continuous bijective function $\mu: \mathbb{R}_{+} \rightarrow \mathbb{R}_{+}$such that

$$
y \circ \mu=\psi \circ z \text {. }
$$

Proof. For any initial condition in $H$, we have $F(\phi(z(t))) \leq \lambda \mathbf{1}$. This is clearly true if $z(t) \in H$. If $z(t) \notin H$ then $z(t) \in \Delta \cap H^{\mathrm{c}}$, by Lemma 4. Thus, $F(z(t))=\left(\lambda-1 / V \mathrm{e}^{-1}\right) \mathbf{1} \leq$ $\lambda$ 1. It follows that $|z(t)|=\sum_{j=1}^{K} z_{j}(t) \leq K \lambda t+\sum_{j=1}^{K} z_{j}(0)$.

Suppose now that $z(t) \in H$ for all $t$. Then the integral $\int_{0}^{\infty}\left(\sum_{j=1}^{K} z_{j}(s)\right)^{-1} \mathrm{~d} s$ diverges. By the intermediate value theorem we deduce that there exists an increasing continuous function $v$ such that

$$
\int_{0}^{v(t)} \frac{\mathrm{d} s}{\sum_{j=1}^{K} z_{j}(s)}=t \quad \text { for all } t \geq 0
$$

In particular,

$$
v^{\prime}(t)=\sum_{j=1}^{K} z_{j}(v(t))
$$

Let $w=\psi \circ z \circ v$, with $w(0)=\psi(w(0))=y(0)$. We have

$$
w^{\prime}(t)=\left.v^{\prime}(t) \frac{\mathrm{d}}{\mathrm{d} s} \psi(z(s))\right|_{s=v(t)}=\left(\sum_{j=1}^{K} z_{j}(v(t))\right) D \psi(z(v(t))) F(w(t)) .
$$


The function $\psi$ is homogeneous of order 0 and, thus, $D \psi(c z)=c^{-1} D \psi(z)$ for all $c>0$. Then

$$
w^{\prime}(t)=D \psi\left(\frac{z(v(t))}{\sum_{j=1}^{n} z_{j}(v(t))}\right) F(w(t))=G(w(t)) .
$$

The solution of the differential equation is unique; therefore, $w(t)=y(t)$. The lemma is proved with $\mu=v^{-1}$.

6.2.3. Local stability of $\psi \circ z$. Clearly, $y_{0}=\mathbf{1} / K$ is an equilibrium point of (21). In the next lemma we prove that this equilibrium is locally stable.

Lemma 12. If $\lambda>\left(\mathrm{e}^{-1} / V\right)\left(1-\gamma^{2} / V^{2}\right)$, there exists $a u>0$ such that, for all solutions $t \mapsto y(t)$ of (21) with $\left|y(0)-y_{0}\right|<u$,

$$
\lim _{t \rightarrow+\infty} \sup _{\left\{y(0) \in \Sigma:\left|y(0)-y_{0}\right|<u\right\}}\left|y(t)-\frac{\mathbf{1}}{V}\right|=0 .
$$

Proof. We denote by $\left.D \alpha(y)\right|_{T_{y}(\Sigma)}$ the differential of $\alpha$ at $y$ restricted to the $(K-1)$ dimensional subspace $T_{y}(\Sigma)$. It is known that if all the eigenvalues of $\left.D \alpha\left(y_{0}\right)\right|_{T_{y}(\Sigma)}$ have a negative real part, the local stability follows (see, e.g. [2, Theorem 1.1]). Let $D^{2} \psi(y)(\cdot, \cdot)$ denote the second differential of $\psi$ at $y$, seen as a bilinear mapping. We have

$$
D \alpha(y)=D^{2} \psi(y)(F(\phi(y)), \cdot)+D \psi(y) D(F \circ \phi)(y) .
$$

The first term in $(23)$ is a matrix and its $(i, j)$ th entry is equal to

$$
\sum_{k=1}^{K} \frac{\partial^{2} \psi(y)_{i}}{\partial y_{j} \partial y_{k}} F(\phi(y))_{k}
$$

Clearly, $F\left(\phi\left(y_{0}\right)\right)=\left(\lambda-\mathrm{e}^{-1} / V\right) \mathbf{1}$. Then a straightforward computation gives

$$
D^{2} \psi\left(y_{0}\right)\left(F\left(\phi\left(y_{0}\right)\right), \cdot\right)=\left(\lambda-\frac{\mathrm{e}^{-1}}{V}\right)(J-K E),
$$

where $E$ is the identity matrix and $J$ is the matrix with all its entries equal to 1 . We also have $D \psi\left(y_{0}\right)=E-J / K$. Finally, (23) can be rewritten as

$$
D \alpha\left(y_{0}\right)=\left(E-\frac{J}{K}\right)\left(D(F \circ \phi)\left(y_{0}\right)-\left(\lambda-\frac{\mathrm{e}^{-1}}{V}\right) E\right) .
$$

The matrix $(E-J / K)$ commutes with all symmetric matrices and has two eigenvalues: eigenvalue 1 (with multiplicity $K-1$ ) and eigenvalue 0 (with multiplicity 1 , associated to the eigenvector 1). By Lemma 10, the eigenvalues of $D(F \circ \phi)\left(y_{0}\right)-\left(\lambda-\mathrm{e}^{-1} / V\right) E$ are

$$
\mu_{i}=-\frac{\mathrm{e}^{-1}\left(V-v_{K-i}\right)^{2}}{V^{3}}-\lambda+\frac{\mathrm{e}^{-1}}{V} \text { for } 0 \leq i \leq K-1 .
$$

The eigenvector associated to $\mu_{0}=\lambda-\mathrm{e}^{-1} / V$ is $\mathbf{1}$. Thus, we have proved that $\lambda-\mathrm{e}^{-1} / V$ is an eigenvalue of multiplicity 1 for $D \alpha\left(y_{0}\right)$ and that the other eigenvalues are $\left(\mu_{i}\right)_{i \geq 1}$. These eigenvalues have negative real parts if and only if $\mu_{1}=-\mathrm{e}^{-1} \gamma^{2} / V^{3}-\lambda+\mathrm{e}^{-1} / V<0$, which is equivalent to $\lambda>\mathrm{e}^{-1}\left(1-\gamma^{2} / V^{2}\right) / V$. The vector space generated by the associated eigenvectors is precisely the tangent hyperplane $T_{y_{0}}(\Sigma)=\mathbf{1}^{\perp}$, the hyperplane orthogonal to 1 . 
Now we can prove Theorem 7. Let $|z(0)| \in C_{u}$ and $y(0)=z(0) /|z(0)|$. Then, by Lemmas 11 and 12 ,

$$
\lim _{t \rightarrow+\infty} \psi(z(t))=\lim _{t \rightarrow+\infty} y(\mu(t))=\frac{1}{K} .
$$

In particular, $\phi(z(t))$ tends towards $\mathbf{1} / V$ as $t$ tends to $\infty$.

\section{Absence of attraction to the diagonal in one particular case}

It has already been pointed out in Section 1.3 and in the previous section that if $\lambda$ is too small, the main diagonal may not be locally stable. In this section we present an example of a graph with locally stable sets of parameters that do not belong to the main diagonal if $\lambda$ is sufficiently small.

Consider a graph $g$ with four vertices placed on a circle. Number the vertices $1,2,3$, and 4 clockwise and assume that each vertex is linked with its two neighbours (so that, for example, vertex 1 has links with 2 and 4). In this case, $K=4$ and $V=3$.

For the fluid limits associated with this graph, consider (21). It can be rewritten in the form

$$
y_{i}^{\prime}(t)=\left(\lambda-\varphi_{i}(t) \exp \left\{-\sum_{j \in V_{i}} \varphi_{j}(t)\right\}\right)-y_{i} \sum_{k=1}^{K}\left(\lambda-\varphi_{k}(t) \exp \left\{-\sum_{j \in V_{k}} \varphi_{j}(t)\right\}\right)
$$

for $i=1, \ldots, K$.

We are interested in the so-called stable points of the latter system of differential equations, i.e. the points for which the right-hand sides of all the equations above are identically 0 . So, if $y(0)$ is such a point, $y(t)$ stays at this point for all $t \geq 0$. Clearly, one stable point is $(1 / K, \ldots, 1 / K)$, which corresponds to the diagonal. However, if $\lambda<\left(\mathrm{e}^{-1} / V\right)\left(1-\gamma^{2} / V^{2}\right)$ $\left(=5 / 27 \mathrm{e}^{-1}\right.$ in our case $)$ then there exist other stable points.

Take $y_{1}(0)=y_{2}(0)$ and $y_{3}(0)=y_{4}(0)$. Since $y_{1}(0)+y_{2}(0)+y_{3}(0)+y_{4}(0)=1$, the equality $y_{3}(0)=\left(1-2 y_{1}(0)\right) / 2$ holds, and the system of differential equations at time $t=0$ reduces to just one (i.e. any) of them. We can show that, for any $\lambda<5 / 27 \mathrm{e}^{-1}$, the right-hand side of this equation equals 0 at three different points: at $\overline{y^{(1)}}=\left(\frac{1}{4}, \frac{1}{4}, \frac{1}{4}, \frac{1}{4}\right)$ and at two others, say $\overline{y^{(2)}}$ and $y^{(3)}$. We can find approximate values of these points numerically. For instance, if $\lambda=0.001$ then $\overline{y^{(2)}} \approx(0.01,0.01,0.49,0.49)$ and $\overline{y^{(3)}} \approx(0.49,0.49,0.01,0.01)$. Numerical results also show that these points are locally stable.

\section{Extensions of the model}

\subsection{Random neighbourhood}

In this subsection we consider a possible extension of our model. Assume that there is a fixed number of points $1, \ldots, K$ and a set of undirected graphs $\left\{g^{j}\right\}_{j=1}^{L}$ each having points $1, \ldots, K$ as their vertices. Assume that at each time $n$ the neighbourhood relations are given by the graph $g^{\eta_{n}}$, where the $\eta_{n}$ are i.i.d. random variables taking the value $j$ with probability $p_{j}$. The need to consider such a variability of neigbourhood relations may be justified by, for instance, the fact that a change of environmental conditions may lead to a change of the radius and/or direction of interactions.

Denote by $\mathcal{V}_{i}^{j}$ the neighbourhood of the point $i$ in the graph $g^{j}$ and by $V_{i}^{j}$ its cardinality. We again assume that the system is regular in the sense that $\mathrm{E} V_{i}^{\eta_{1}}=V$ for all $i$. 
Following the proof of Theorem 3, we can show that the fluid limits of the model described above satisfy the following differential equation:

$$
z_{i}^{\prime}(t)=\lambda-\sum_{k=1}^{L} p_{k} \varphi_{i}^{k}(t) \exp \left\{-\sum_{j \in V_{i}^{k}} \varphi_{j}^{k}(t)\right\},
$$

where the $\varphi_{i}^{k}(t)$ are defined in a natural way. Using the same methods as those used in the proof of Theorem 2, it can be shown that the system with random neighbourhood is stable if $\lambda<\mathrm{e}^{-1} / V$.

\subsection{Nonregular graphs with space-inhomogeneous input}

Although Remark 3 provides sufficient conditions for stability in this case, the conditions are not easy to verify. Here we give some other conditions that are also sufficient for the stability of the system. Assume now that $\mathrm{E} V_{i}^{\eta_{1}}=V_{i}$, the $V_{i}$ are not necessarily equal. Set $V=\max _{i} V_{i}$. Assume also that $\mathrm{E} \xi_{i}^{n}=\lambda_{i}$, so that the input is 'space inhomogeneous'. Set $\lambda=\max _{i} \lambda_{i}$. Clearly, all the results concerning fluid limits also hold in this case, and it is easy to see that we can prove the following result.

Theorem 8. The system described above is stable provided $\lambda<\mathrm{e}^{-1} / V$.

\section{Acknowledgements}

The authors are grateful to F. Baccelli and D. Denisov for useful discussions on different parts of this work, to A. Stolyar for bringing his paper [18] to their attention, and to A. Richards for careful reading and important comments.

The authors would also like to thank Framework 7 EURO NF project for travel support. The second author acknowledges travel support from EPSRC grant number R58765/01.

\section{References}

[1] Abramson, N. (1970). The ALOHA system - another alternative for computer communications. AFIPS Conf. Proc. 36, 295-298.

[2] Coddington, E. A. And Levinson, N. (1955). Theory of Ordinary Differential Equations. McGraw-Hill, New York.

[3] DAI, J. G. (1995). On positive Harris recurrence of multiclass queueing networks: a unified approach via fluid limits. Ann. Appl. Prob. 5, 49-77.

[4] Dai, J. G. AND MeYn, S. P. (1995). Stability and convergence of moments for multiclass queueing networks via fluid limit models. IEEE Trans. Automatic Control 40, 1889-1904.

[5] Douc, R., Fort, G., Moulines, E. And Soulier, P. (2004). Practical drift conditions for subgeometric rates of convergence. Ann. Appl. Prob. 3, 1353-1377.

[6] EPHREMides, A. AND HAJEK, B. (1998). Information theory and communication networks: an unconsummated union. IEEE Trans. Inf. Theory 44, 2416-2434.

[7] Ephremides, A., Nguyen, G. B. and Wieselthier, J. E. (2005). Random access in overlapping cells. In Proc. 11th European Wireless Conf. (Cyprus, April 2005), pp. 659-665.

[8] Feller, W. (1971). An Introduction to Probability Theory and Its Applications, Vol. 2, 2nd edn. John Wiley, New York.

[9] Foss, S. G. (1992). Stochastic recursive sequences and their applications in queueing. Doctoral Thesis, Institute of Mathematics, Novosibirsk, Russia.

[10] Foss, S. AND KovalevskiI, A. (1999). A stability criterion via fluid limits and its application to a polling system. Queueing Systems 32, 131-168.

[11] HajeK, B. (1982). Hitting-time and occupation-time bounds implied by drift analysis with applications. $A d v$. Appl. Prob. 14, 502-525.

[12] Meyn, S. P. (1995). Transience of multiclass queueing networks via fluid limit models. Ann. Appl. Prob. 5, 946-957. 
[13] Meyn, S. P. And Tweedie, R. L. (1993). Markov Chains and Stochastic Stability. Springer, London.

[14] Mikhal̆Lov, V. (1988). Geometric analysis of the stability of Markov chains in $R_{+}^{n}$ and its application to the calculation of the capacity of an adaptive random multiple access algorithm. Problems Inform. Transmission 24, 47-56.

[15] Roberts, L. G. (1975). ALOHA packet system with and without slots and capture. Comput. Commun. Rev. 5, $28-42$.

[16] Seneta, E. (1981). Nonnegative Matrices and Markov Chains. Springer, New York.

[17] Stolyar, A. L. (1995). On the stability of multiclass queueing networks: a relaxed sufficient condition via limiting fluid processes. Markov Process. Relat. Fields 1, 491-512.

[18] Stolyar, A. L. (2008). Dynamic distributed scheduling in random access networks. J. Appl. Prob. 45, $297-313$. 